\title{
Neue Mukosa für Colitis-Patienten
}

Die Barrierestörung der Darmmukosa beheben - dies wird bei Colitis ulcerosa zukünftig ein neuer Therapieansatz sein.

Im Verlauf einer Colitis ulcerosa spielt die Barrierestörung schon zu Beginn eine große Rolle. Die Mukosa wird im Verlauf einer Colitis ulcerosa, beginnend im Rektum, nach und nach zerstört bis hin zur Pankolitis mit großflächiger Entzündung und Vernarbungsprozessen. Dies ist ein Grund, warum mit der üblichen Therapie, beginnend mit 5-Aminosalizylaten über Steroide bis hin zu Immuntherapeutika, vielen Patienten nicht oder nicht ausreichend geholfen werden kann. Mit Phosphatidylcholin werde man nun eine „völlig neue Therapieschiene" an die Hand bekommen, sagte Prof. Dr. Herbert Tilg, Gastroenterologe aus Innsbruck.

Phosphatidylcholin ist ein Hauptbestandteil der Schleimschicht. Bei Colitis-
ulcerosa-Patienten ist der Phosphatidylgehalt in der Mukosa deutlich vermindert. Dadurch gelangen Bakterien und Fremdstoffe in direkten Kontakt mit der Darmwand und dies löst entsprechende immunologische Reaktionen und Entzündungen aus. In Studien wird seit einiger Zeit versucht, Phosphatidylcholin mittels innovativer galenischer Formulierungen gezielt dem Dickdarm zuzuführen und auf diese Weise den Schleimhautschutz wieder zu verbessern. Die bisherigen Ergebnisse sind ermutigend.

Tilg zitierte eine Studie bei 156 CU-Patienten, die unzureichend auf Mesalazin ansprachen, unter blutigen Durchfällen litten und einen Krankheitsaktivitätsscore (SCCAI - Simple Clinical Colitis Activity Index) von mindesten 5 aufwiesen. Die Studienteilnehmer hatten randomisiert und doppelblind ihre Standardtherapie plus Placebo erhalten oder retardiertes Phosphotidylcholin LT-02 in verschiedenen Do- sierungen. In der Placebo-Gruppe sank der SCCAI um $33,3 \%$ von 9,0 auf 6,0 Punkte, unter $0,8 \mathrm{~g}$ LT- 02 von 8,8 auf 4,9 Punkte $(44,3 \%)$ und unter der höchsten Dosis von 3,2 g LT-02 von 8,4 auf 4,1 Punkte (51,7\%) - der Unterschied zu Placebo war signifikant.

Eine Mukosaheilung war bei jedem dritten Patienten unter Placebo und fast der Hälfte der LT-02-behandelten Patienten beobachtet worden. Für die Patienten spürbare Verbesserungen machten sich in der Verumgruppe zwei Wochen früher bemerkbar, als in der Kontrollgruppe (Karner $\mathrm{M}$ et al. Am J Gastroenterol 2014;109:1041-51). Nach Angaben von Tilg laufen derzeit zwei Phase-III-Studien, um die Wirksamkeit dieses Therapieprinzips zu sichern.

(TM)

Symposium 201. Gut-liver interactions: from IBD to NASH. 11.-12. März 2016 in Innsbruck. Veranstaltung: Falk Foundation e.V..

\section{Renaissance der Gallensäuren}

\section{Die Fibrose der Leber aufhalten}

\begin{abstract}
Neue Einblicke in die Wirkmechanismen der Gallensäuren haben zu einem Forschungsschub in therapeutischen Anwendung geführt.
\end{abstract}

Gallensäuren sind enterohepatische Hormone. Über sie erhalten Zellen Informationen über den Ernährungszustand, sie sind an der Regulation des Fettstoffwechsels sowie der Glukose- und Energiehomöostase beteiligt. Außerdem sind sie immunmodulierend und bakteriostatisch: „Daher können sie als ein Teil des Immunsystems des Darms angesehen werden“, er- klärte Prof. Dr. Michael Trauner, Wien. Dies hat Bedeutung für die Behandlung immunologischer Lebererkrankungen.

Die Inzidenz und Prävalenz der primären biliaren Cholangitis (PBC) habe sich in den letzten zehn Jahren verdoppelt, so Trauner. Wegen der frühen Diagnose verläuft sie heute oft mild und muss nicht notgedrungen zur Zirrhose führen. Mit Ursodesoxycholsäure (UDCA, z.B. Ursofalk $^{\star}$ ) kann die Fibrose aufgehalten werden. Sie wird in einer Dosis von 13 bis 15 $\mathrm{mg} / \mathrm{d}$ allen PBC-Patienten mit abnormen Leberwerten empfohlen.

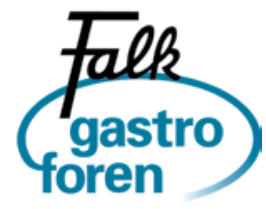

Besuchen Sie das nächste Falk Gastro-Forum „Viszeralmedizin heute: von der Empirie zur personalisierten Medizin" am Samstag, den 4. Juni 2016 in Essen; Infos: www.fortbildung@falkfoundation.de

Gallensäuren gewinnen jedoch auch an Bedeutung für die Therapie metabolischer Erkrankungen wie Fettleber, Diabetes mellitus und Adipositias. Studien hierzu laufen. Chemisch modifizierte UDCA-Derivate wie Nor-Ursodesoxycholsäure (norUDCA) und Gallensäuren-SignalingMimetika werden bereits klinisch angewendet. So wurden Frauen mit PBC, die nicht auf UDCA ansprachen, erfolgreich mit dem FXR-Liganden Obeticholsäure behandelt. Die Publikation der Daten wird demnächst erwartet.

NorUDCA zeigte zudem bei primär sklerosierender Cholangitis (PSC) anticholestatische, antiinflammatorische und antifibrotische Effekte. Eine Phase-IIIStudie hierzu ist geplant.

(TM) 